\title{
Hábitos alimentarios y condición física en estudiantes de pedagogía en educación física
}

\author{
Eating habits and physical condition \\ of physical education students
}

\begin{abstract}
Objective: To determinate dietary habits and physical condition of physical activity students from the Autonomous University of Chile, Temuco, relating these to their fitness level. Methods: An observational cross-sectional study was conducted among 239 students (76.5\% males). Assessment included a food questionnaire, anthropometric measurements and physical condition. Results: In a sample of 239 students, $35.6 \%$ of them were overweight or obese as well as having unhealthy dietary habits independent of the academic year at university. Only $4.7 \%$ of the students used the national recommendations for fruit intake and only $30 \%$ ate breakfast daily. In men weight, waist circumference and BMI were negatively associated with the Nvette Course test $(R=-0.203, R=-0.249$ and $R=-0.196, p<0.01$ respectively). Conclusion: Physical activity education students have poor dietary habits throughout the university academic years while their fitness decreases irregularly as studies progress. This is a major concern since they will become healthy life style role models for their future students. .

Key words: University student, anthropometry, fitness, food survey.
\end{abstract}

Samuel Durán A. (1)

Pablo Valdés $B$. $(2,3)$

Andrés Godoy C. (2)

Tomás Herrera V. $(4,5)$

(1) Facultad de Ciencias de la Salud, Universidad San Sebastián, Chile. (2) Departamento de Educación Física, Facultad de Educación, Universidad Autónoma de Chile, sede Temuco, Chile. (3) Centro de Deportes y Salud, Universidad Autónoma de Chile, sede Temuco, Chile. Universidad Autónoma de Chile, sede Temuco, Chile.
(4) Facultad de Ciencias Médicas de la Universidad de Santiago de Chile, Santiago, Chile

(5) Facultad de Ciencias de la Actividad Física, Universidad San Sebastián, Santiago, Chile.

Dirigir la correspondencia a: Profesor

Samuel Durán Agüero Universidad San Sebastián Chile

Lota 2465

Providencia,

Santiago, Chile

Teléfono: +56984335892

E-mail:samuel.duran@uss.cl

Este trabajo fue recibido el 19 de Mayo de 2014 y aceptado para ser publicado el 22 de Julio de 2014.

\section{INTRODUCCIÓN}

Es indudable que los avances tecnológicos han brindado mayor confort en el quehacer de las personas; sin embargo, los cambios en el estilo de vida también han generado hábitos alimentarios y de actividad física poco saludable (1-5). En Chile esta tendencia es creciente, reflejándose en la alta prevalencia de sedentarismo que alcanza un $82.7 \%$ de la población mayor de 18 años y en los altos niveles de sobrepeso y obesidad que evidencian los niños y niñas de octavo año básico (48\%) (5).

En este contexto, diversos han sido los trabajos que dan cuenta de los hábitos alimentarios de estudiantes, en especial universitarios, por considerarlos una población susceptible de adquirir costumbres poco saludables, debido a encontrarse en un periodo sujeto a constantes cambios como es; largas horas de estudio, clases en horarios diversos, aumento de la vida nocturna, escases de presupuesto, entre otros; acciones que redundan en ayunos prolongados, saltarse comidas y consumo de alimentos ricos en grasa (1-3, 6-12).

Existen estudios que exponen los resultados de estudiantes universitarios, tanto nacionales como internacionales relativos a la condición física, en ellos se puede apreciar mejores puntajes que el resto de los ciudadanos chilenos $(4,5)$, en particular aquellos que aportan datos concernientes a estudiantes de Pedagogía en Educación Física, quienes manifiestan hábitos de actividad física más saludables respecto a la población general (13-17).

El Ministerio de Educación de Chile (MINEDUC), ha realizado recientes transformaciones en la asignatura de educación física, todo ello tras conocer los malos resultados que obtuvieron los alumnos y alumnas en las pruebas de antropometría y condición física realizadas durante los años 2011 y 2012 (5), reestructurando su contenido y vinculándola hacia la vida activa y la salud (18). En este sentido los cambios sociales y curriculares vividos en Chile, obligan a estudiar variables relacionadas con los futuros profesores de educación física, ya que son los encargados de fomentar y educar en torno a los hábitos de vida saludable al interior de los establecimientos educacionales, y para conseguir dicho propósito es importante fortalecer y cultivar las conductas adecuadas durante su formación profesional.

En este sentido, la presente investigación tiene por objetivo determinar los hábitos alimentarios y condición física 
de los estudiantes de Pedagogía en Educación Física de la Universidad Autónoma de Chile, sede Temuco.

\section{SUJETOS Y MÉTODOS}

El tipo de investigación contempla un diseño no experimental transversal correlacional, con un enfoque cuantitativo.

\section{Sujetos}

La población estuvo constituida por todos los alumnos y alumnas de la Carrera de Pedagogía en Educación Física de la Universidad Autónoma de Chile (UA), sede Temuco $(n=420)$. La muestra fue seleccionada bajo un criterio no probabilístico, que incluyó a 239 estudiantes (56,9\%), distribuidos en mujeres $(n=56)$ y hombres $(n=183)$, quienes se encuentran en el rango de 18 a 31 años, alcanzando un promedio de edad de $21,5 \pm 2,1$ años.

En relación al peso y la talla, el promedio fue $62,7 \pm 8,1$ kg y 1,63 $\pm 0,05$ metros para las mujeres, y de 73,9 $\pm 10,8 \mathrm{~kg}$ y 1,74 $\pm 0,05$ metros para los hombres. Se incluyó a todos los estudiantes de la carrera desde la cohorte 2013 hasta 2009, que se encontraban presentes al momento de realizar las evaluaciones; además debían cumplir con la firma de un consentimiento informado, excluyendo a quienes no asistieron, presentaron licencia médica o no firmaran el documento solicitado. El estudio fue desarrollado siguiendo lo expuesto en la Declaración de Helsinki, respecto al trabajo con seres humanos y aprobado por el Comité de Etica de la Universidad San Sebastian.

\section{Encuesta}

El instrumento utilizado para la recolección de datos, correspondió a una encuesta denominada "Encuesta sobre hábitos alimentarios" creada por Durán y cols. Se realizó una validación del cuestionario por juicio de 25 expertos, realizada a través del método Delphi (19). El juicio de experto permitió contrastar cada uno de los ítems con la teoría en relación a los hábitos alimentarios de los estudiantes. La encuesta tiene por objetivo medir los hábitos alimentarios de los sujetos y se compone de dos ámbitos, de auto aplicación, el primero compuesto por nueve ítems con un puntaje mínimo de 1 y máximo de 5 por pregunta (escala de tipo Likert), que indica la frecuencia de hábitos saludables (consumo de desayuno, cena y comida cacera) como la frecuencia de consumo de grupos de alimentos recomendados por las guías alimentarias chilenas (lácteos, frutas, verduras, leguminosas, pescados y cereales integrales), que va desde no consume (1 punto), hasta las porciones día/semana sugeridas (5 puntos) obteniendo una calificación de las respuestas que varía de 9 a 45 puntos (mayor valor mejores hábitos alimentarios). Mientras que el segundo ámbito consta de seis ítems, alimentos o grupos de alimentos identificados como promotores de enfermedades crónicas no transmisibles (bebidas azucaradas, alcohol, alimentos fritos, comida rápida, bocadillos) y se agregó un hábito alimentario negativo como es adicionar sal a las comidas sin probarlas, cinco preguntas con un puntaje idéntico al anterior 1 (no consume) a 5 (>x porción día/semana) y sólo una calificada de 1 al 3 (sal), alcanzando un valor que va de 6 a 28 puntos (mayor valor peores hábitos alimentarios).

\section{Evaluaciones de condición física}

Para evaluar la condición física de los estudiantes UA; se utilizaron las pruebas descritas en el sistema de medición de la calidad de la educación (SIMCE) de Educación Física instaurado por el MINEDUC (5), quienes señalan que "las pruebas seleccionados han sido validadas y estandarizadas nacional e internacionalmente" (20-28). Dicha evaluación consta de 8 pruebas distribuidas en 4 ámbitos, estos son: 1.- Antropometría: De acuerdo a lo expuesto en el MINEDUC (5) "Hace referencia a los aspectos relacionados con las dimensiones corporales de una persona". 1.1.- Estimación del Índice de masa Corporal (IMC): Esta prueba se utiliza para determinar la relación entre el peso y la talla de las personas. El IMC se obtiene dividiendo el peso por la altura al cuadrado (5). 1.2.- Perímetro de cintura: Esta prueba se utiliza para estimar la acumulación de grasa en la zona central del cuerpo, al dividir el perímetro de cintura por la estatura se tendría la capacidad para predecir factores de riesgo cardiovascular (29). 2.- Rendimiento muscular: De acuerdo a lo expuesto por Lamela y Nogueira y cols.. (30-31) "Hacen referencia a la capacidad de trabajo de los músculos. Dentro de este componente, se evaluó la fuerza y la resistencia muscular". 2.1.- Abdominales cortos: "Esta prueba se utiliza para evaluar la resistencia de la musculatura flexora del tronco" (5). 2.2.- Salto largo a pies juntos: "El objetivo de esta prueba es evaluar la fuerza explosiva del tren inferior" (5). 2.3.- Flexo-extensión de codos: "Esta prueba se utiliza para medir la resistencia de la fuerza del tren superior" (5). 3.- Flexibilidad: "Se define como la capacidad funcional de las articulaciones de moverse en todo su rango de movimiento o bajo la influencia de fuerzas externas, sin dañar músculos ni articulaciones" (30). 3.1.- Flexión de tronco adelante (Wells y Dillon adaptado): "El objetivo de esta prueba es determinar el rango de movimiento de la articulación coxofemoral y de la columna lumbar; determinar la capacidad de elongación de las musculaturas isquiotibial y glútea, y determinar la capacidad flexora de la columna vertebral" (5). 4.- Resistencia cardiorrespiratoria: "Es la capacidad del organismo de suministrar el oxígeno necesario a los músculos y posponer la aparición de la fatiga en una actividad física" (30). 4.1.- Test de Course Navette: Este test se utiliza para evaluar la potencia aeróbica máxima; es decir, la capacidad que tiene el cuerpo para suministrar el oxígeno necesario a los músculos durante un esfuerzo máximo (21).

Se asociaron los puntajes obtenidos por los estudiantes, de acuerdo a los criterios establecidos en la literatura (32-34), para la prueba de salto largo a pies juntos se contemplaron los registros que alcanzaron los niveles de 6 al 10, en una escala de 1 a 10, donde 1 corresponde al nivel más bajo y 10 al más alto; para la prueba de flexo-extensión de codos se consideraron los resultados que alcanzaron los niveles de aceptable, bueno y excelente; para la prueba de flexibilidad se estimaron los puntajes que estuvieron dentro de los niveles aceptable, bueno y excelente; y para la prueba de course navette se consideró los resultados que alcanzaron los niveles de promedio, sobre el promedio y bueno. Se utilizaron las categorías señaladas pero en sus valores numéricos para establecer el nivel de satisfactorio.

\section{Procedimiento}

El proceso para la aplicación de la pruebas, se distribuyó de la siguiente manera: en primer término los estudiantes UA respondieron la encuesta sobre hábitos alimentarios. El peso corporal fue evaluado con una balanza digital (Scale-tronix, USA), la estatura con estadiómetro (Seca modelo 220, Alemania), el perímetro de cintura con cinta métrica (Sanny, Brasil). Los instrumentos ostentan una precisión de $0.1 \mathrm{~kg}, 0.10 \mathrm{~cm}$ y $0.1 \mathrm{~mm}$, respectivamente. En el gimnasio de la Universidad, se realizó un calentamiento general de 15 minutos guiado por un profesor. Posteriormente se dividieron en 4 grupos para realizar las pruebas de: abdominales cortos, salto largo a pies juntos, flexo-extensión de codos y flexión de tronco adelan- 
te; una vez que todos los estudiantes pasaban por todas las estaciones mencionadas, se finalizaba con el test de Course Navette a todo el curso. Las evaluaciones se realizaron previa autorización de la dirección de la carrera de Pedagogía en Educación Física y dentro del periodo comprendido entre el 04 y el 15 de noviembre del 2013.

Análisis estadístico

Los resultados se presentan como promedios \pm desviaciones estándar y como porcentajes según el tipo de variable. Para la comparación de las variables por sexo se utilizó la prueba $\mathrm{t}$ de Student. Se hicieron pruebas de bondad de ajuste y de homogeneidad de varianzas. Para la comparación entre los grupos se utilizó el método de análisis de varianza (ANOVA) y para la estimación de las correlaciones se utilizó el coeficiente de Pearson.

Los resultados fueron obtenidos con el programa estadístico SPSS 19.0 ®. Se utilizó un nivel de significación de 0,05.

\section{RESULTADOS}

En la tabla 1 se aprecia la antropometría, pruebas físicas y encuesta de alimentación de todos los estudiantes UA, expre-

\section{TABLA 1}

Antropometría, pruebas físicas y hábitos alimentarios de los estudiantes de educación física, distribuidos por sexo.

\begin{tabular}{|c|c|c|c|c|}
\hline & $\begin{array}{c}\text { Total } \\
n=239\end{array}$ & $\begin{array}{c}\text { Mujer } \\
n=56\end{array}$ & $\begin{array}{c}\text { Hombre } \\
n=183\end{array}$ & Valor $p$ \\
\hline Edad (años): & $21,5 \pm 2,1$ & $21,1 \pm 2,1$ & $21,7 \pm 2,2$ & 0,06 \\
\hline Peso $(k g)$ & $71,1 \pm 11,3$ & $62,7 \pm 8,1$ & $73,9 \pm 10,8$ & 0,001 \\
\hline Talla (m) & $1,72 \pm 0,07$ & $1,63 \pm 0,05$ & $1,74 \pm 0,05$ & 0,001 \\
\hline $\mathrm{PC}(\mathrm{cm})$ & $77 \pm 9,3$ & $72,7 \pm 6,3$ & $79,6 \pm 9,4$ & 0,001 \\
\hline $\mathrm{IMC}\left(\mathrm{kg} / \mathrm{m}^{2}\right)$ & $23,9 \pm 2,8$ & $23,5 \pm 2,6$ & $24,1 \pm 2,9$ & 0,17 \\
\hline $\mathrm{RCE}(\mathrm{cm})$ & $0,45 \pm 0,04$ & $0,44 \pm 0,03$ & $0,45 \pm 0,05$ & 0,09 \\
\hline Abdominales cortos (rep.) & $24,7 \pm 1,5$ & $24,2 \pm 2,7$ & $24,9 \pm 0,8$ & 0,018 \\
\hline Flexo-extensión de codos (rep.) & $34,5 \pm 6,2$ & $36,2 \pm 5,9$ & $33,9 \pm 6,2$ & 0,017 \\
\hline Salto largo a pies juntos (cm) & $190,8 \pm 31,2$ & $154,3 \pm 21,9$ & $202,9 \pm 23,5$ & 0,001 \\
\hline Flexibilidad (cm) & $35,1 \pm 6,2$ & $36,2 \pm 5,9$ & $33,9 \pm 6,2$ & 0,017 \\
\hline Test de Course Navette (min) & $7,9 \pm 2,2$ & $5,4 \pm 1,8$ & $8,7 \pm 1,6$ & 0,001 \\
\hline Encuesta alimentaria (1) & $26,8 \pm 5,3$ & $25,9 \pm 4,6$ & $26,6 \pm 5,6$ & 0,37 \\
\hline Encuesta alimentaria (2) & $14,7 \pm 3,1$ & $14,1 \pm 2,8$ & $14,9 \pm 3,1$ & 0,10 \\
\hline Delta encuesta & $11,7 \pm 6,3$ & $11,8 \pm 5,4$ & $11,7 \pm 6,5$ & 0,97 \\
\hline Consumo de desayuno & $3,4 \pm 1,3$ & $3,5 \pm 1,4$ & $3,4 \pm 1,3$ & 0,65 \\
\hline Consumo lácteos descremados & $2,5 \pm 0,9$ & $2,4 \pm 1,0$ & $2,6 \pm 0,9$ & 0,14 \\
\hline Consumo de frutas & $2,7 \pm 0,9$ & $2,7 \pm 0,9$ & $2,8 \pm 1,1$ & 0,75 \\
\hline Consumo de verduras & $3,1 \pm 1,1$ & $3,3 \pm 1,1$ & $3,0 \pm 1,1$ & 0,13 \\
\hline Consumo de pescado & $2,6 \pm 1,0$ & $2,5 \pm 0,9$ & $2,6 \pm 1,1$ & 0,31 \\
\hline Consumo de leguminosas & $2,8 \pm 1,0$ & $2,6 \pm 2,5$ & $2,8 \pm 1,1$ & 0,21 \\
\hline Consumo alimentos integrales & $2,1 \pm 1,1$ & $2,1 \pm 1,2$ & $2,1 \pm 1,1$ & 0,81 \\
\hline Consumo de comida casera & $4,0 \pm 1,1$ & $4,1 \pm 1,0$ & $3,9 \pm 1,2$ & 0,39 \\
\hline Consumo de cena & $2,8 \pm 1,4$ & $2,4 \pm 1,1$ & $2,9 \pm 1,3$ & 0,008 \\
\hline Consumo de bebidas azucaradas & $3,3 \pm 1,1$ & $3,0 \pm 1,1$ & $3,3 \pm 1,2$ & 0,09 \\
\hline Consumo de alcohol & $2,2 \pm 1,2$ & $2,0 \pm 1,1$ & $2,2 \pm 1,2$ & 0,22 \\
\hline Consumo de frituras & $2,8 \pm 1,1$ & $2,5 \pm 1,0$ & $2,9 \pm 2,5$ & 0,031 \\
\hline Consumo de sal & $1,7 \pm 0,7$ & $1,7 \pm 0,7$ & $1,7 \pm 0,7$ & 0,98 \\
\hline Consumo de comida rápida & $2,1 \pm 0,7$ & $2,0 \pm 0,8$ & $2,2 \pm 0,6$ & 0,21 \\
\hline Consumo de snacks dulces & $2,4 \pm 0,9$ & $2,6 \pm 0,8$ & $2,3 \pm 0,9$ & 0,02 \\
\hline
\end{tabular}

Valores expresados en media $\pm D E$, Prueba $T$ de Student. $P C=$ Perímetro de Cintura, $I M C=$ índice de masa corporal, $R C E=$ razón cintura estatura, rep.= repeticiones. 


\section{TABLA 2}

Antropometría, pruebas físicas y hábitos alimentarios en estudiantes de sexo femenino de educación física, distribuidas por cohorte.

\begin{tabular}{|c|c|c|c|c|c|}
\hline & $\begin{array}{c}2009 \\
n=6\end{array}$ & $\begin{array}{c}2010 \\
n=7\end{array}$ & $\begin{array}{l}2011 \\
n=14\end{array}$ & $\begin{array}{l}2012 \\
n=16\end{array}$ & $\begin{array}{l}2013 \\
n=15\end{array}$ \\
\hline Edad (años): & $24,0 \pm 0,6^{a, b, c}$ & $23,2 \pm 1,7^{d, e, f}$ & $21,5 \pm 1,5^{\mathrm{a}, \mathrm{d}, \mathrm{g}, \mathrm{h}}$ & $20,0 \pm 0,6^{\mathrm{b}, \mathrm{e}, \mathrm{g}}$ & $19,4 \pm 1,9^{c, f, h}$ \\
\hline Peso (kg) & $61,9 \pm 8,5$ & $67,9 \pm 9,6$ & $63,1 \pm 8,8$ & $59,1 \pm 6,7$ & $61,8 \pm 7,9$ \\
\hline Talla (m) & $1,59 \pm 0,04^{a}$ & $1,62 \pm 0,05$ & $1,62 \pm 0,04$ & $1,61 \pm 0,04$ & $1,65 \pm 0,03^{a}$ \\
\hline $\mathrm{PC}(\mathrm{cm})$ & $72,0 \pm 5,4$ & $73,7 \pm 7,4$ & $73,5 \pm 6,5$ & $69,0 \pm 7,1$ & $72,9 \pm 6,0$ \\
\hline $\mathrm{IMC}\left(\mathrm{kg} / \mathrm{mt}^{2}\right)$ & $24,3 \pm 3,2$ & $25,7 \pm 2,9$ & $23,7 \pm 2,9$ & $22,4 \pm 1,8$ & $22,6 \pm 2,5$ \\
\hline RCE (cm) & $0,45 \pm 0,04$ & $0,45 \pm 0,05$ & $0,44 \pm 0,03$ & $0,42 \pm 0,04$ & $0,44 \pm 0,03$ \\
\hline Abdominal cortos (rep.) & $25,0 \pm 0,0$ & $25,0 \pm 0,0$ & $23,1 \pm 4,7$ & $25,0 \pm 0,0$ & $25,0 \pm 0,0$ \\
\hline Flexo-extensión de codos (rep.) & $38,5 \pm 4,1$ & $36,5 \pm 2,9$ & $34,5 \pm 5,4$ & $36,5 \pm 6,9$ & $36,4 \pm 6,9$ \\
\hline Salto largo a pies juntos (cm) & $153,8 \pm 10,1$ & $165,2 \pm 22,2$ & $154,2 \pm 24,0$ & $155,0 \pm 18,2$ & $118,3 \pm 66,1$ \\
\hline Flexibilidad (cm): & $38,5 \pm 4,1$ & $36,5 \pm 2,9$ & $34,5 \pm 5,4$ & $36,5 \pm 6,9$ & $37,6 \pm 6,8$ \\
\hline Test de Course Navette (min) & $4,0 \pm 2,9$ & $5,2 \pm 1,0$ & $5,1 \pm 1,7$ & $6,8 \pm 1,8$ & $5,5 \pm 1,6$ \\
\hline Encuesta alimentaria (1) & $25,8 \pm 6,2$ & $27,2 \pm 4,9$ & $25,1 \pm 3,8$ & $26,0 \pm 5,1$ & $25,9 \pm 4,2$ \\
\hline Encuesta alimentaria (2) & $13,6 \pm 2,1$ & $12,1 \pm 1,4$ & $14,6 \pm 3,8$ & $14,1 \pm 2,6$ & $14,8 \pm 2,6$ \\
\hline Delta & $9,1 \pm 5,0$ & $12,8 \pm 6,6$ & $12,7 \pm 6,0$ & $9,6 \pm 6,8$ & $12,3 \pm 6,9$ \\
\hline
\end{tabular}

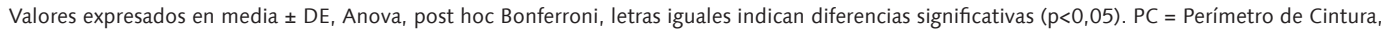
$I M C=$ índice de masa corporal, RCE= razón cintura estatura, rep.= repeticiones.

TABLA 3

Antropometría, pruebas físicas y hábitos alimentarios en estudiantes de sexo masculino de educación física, distribuidos por cohorte.

\begin{tabular}{|c|c|c|c|c|c|}
\hline & $\begin{array}{l}2009 \\
n=17\end{array}$ & $\begin{array}{l}2010 \\
n=46\end{array}$ & $\begin{array}{l}2011 \\
n=36\end{array}$ & $\begin{array}{l}2012 \\
n=28\end{array}$ & $\begin{array}{l}2013 \\
n=39\end{array}$ \\
\hline Edad (años): & $24,2 \pm 1,5^{a, b, c}$ & $23,0 \pm 1,5^{d, e, f}$ & $21,8 \pm 1,9^{a, d, g, h}$ & $20,6 \pm 1,4^{b, e, g}$ & $19,7 \pm 1,6^{c, f, h}$ \\
\hline Peso (kg) & $75,5 \pm 6,0$ & $74,2 \pm 9,6$ & $75,9 \pm 13,9$ & $71,6 \pm 9,4$ & $72,6 \pm 12,1$ \\
\hline Talla (m) & $1,72 \pm 0,04$ & $1,75 \pm 0,05$ & $1,76 \pm 0,06$ & $1,74 \pm 0,05$ & $1,73 \pm 0,05$ \\
\hline $\mathrm{PC}(\mathrm{cm})$ & $81,6 \pm 5,3$ & $79,7 \pm 7,5$ & $82,2 \pm 9,2$ & $77,1 \pm 10,5$ & $77,8 \pm 11,9$ \\
\hline $\mathrm{IMC}\left(\mathrm{kg} / \mathrm{mt}^{2}\right)$ & $25,3 \pm 1,7$ & $24,0 \pm 2,5$ & $24,3 \pm 3,5$ & $23,4 \pm 2,6$ & $23,9 \pm 3,2$ \\
\hline RCE (cm) & $0,47 \pm 0,03$ & $0,45 \pm 0,04$ & $0,46 \pm 0,04$ & $0,44 \pm 0,05$ & $0,44 \pm 0,06$ \\
\hline Abdominales cortos (rep.) & $25,0 \pm 0,0$ & $25,0 \pm 0,0$ & $25,0 \pm 0,0$ & $24,6 \pm 1,9$ & $24,9 \pm 0,1$ \\
\hline Flexo-extensión de codos (rep.) & $30,0 \pm 4,3^{a, b}$ & $35,7 \pm 6,4^{a}$ & $33,5 \pm 5,3$ & $33,5 \pm 6,5$ & $35,1 \pm 6,6^{b}$ \\
\hline Salto largo a pies juntos (cm) & $190,5 \pm 17,2^{a, b}$ & $211,0 \pm 20,1$ & $211,9 \pm 26,2^{b, d}$ & $201,3 \pm 22,5$ & $191,5 \pm 22,2^{c, d}$ \\
\hline Flexibilidad (cm) & $30,0 \pm 4,3 a, b$ & $35,2 \pm 6,5^{a}$ & $33,5 \pm 5,3$ & $33,5 \pm 6,5$ & $35,2 \pm 5,7^{b}$ \\
\hline Test de Course Navette (min) & $8,5 \pm 1,8$ & $9,3 \pm 1,6$ & $8,5 \pm 1,6$ & $8,3 \pm 1,8$ & $8,7 \pm 1,7$ \\
\hline Encuesta alimentaria (1) & $23,6 \pm 3,3$ & $27,0 \pm 5,9$ & $27,6 \pm 5,4$ & $25,1 \pm 6,1$ & $27,7 \pm 5,5$ \\
\hline Encuesta alimentaria (2) & $14,5 \pm 3,1$ & $14,2 \pm 3,1$ & $14,8 \pm 2,7$ & $15,5 \pm 3,7$ & $15,4 \pm 3,1$ \\
\hline Delta & $12,1 \pm 5,8$ & $15,1 \pm 4,7$ & $10,5 \pm 5,7$ & $11,8 \pm 6,0$ & $11,1 \pm 5,0$ \\
\hline
\end{tabular}

Valores expresados en media $\pm D E$, Anova, post hoc Bonferroni, letras similares indica diferencias significativas $(p<0,05)$. PC $=$ Perímetro de Cintura, $\mathrm{IMC}=$ índice de masa corporal, $\mathrm{RCE}=$ razón cintura estatura, rep.= repeticiones. 
sados en media y desviación estándar (DE). Las pruebas físicas de los hombres UA presentaron puntajes significativamente superiores en todas las evaluaciones que las mujeres UA, mientras que en la encuesta de alimentación se observaron diferencias en el consumo de cena y alimentos fritos, siendo significativamente mayor en los hombres UA, las mujeres UA exhibieron un mayor consumo de bocadillos dulces. En la encuesta de hábitos alimentarios, tanto los aspectos positivos como negativos se encontraron en la media todos los estudiantes UA.

En relación a la distribución por sexo se puede apreciar en la tabla 2 los valores alcanzados por las mujeres UA por cohorte (año de ingreso a la Universidad), presentando diferencias en edad y talla. En la tabla 3, se observan los registros conseguidos por los varones UA, quienes exhiben diferencias en edad, salto largo a pies juntos y flexibilidad.

Al comparar los resultados en las pruebas de condición física de los estudiantes UA por cohorte, se advierte en las tablas 2 y 3 que para las pruebas de flexibilidad y abdominales cortos, no existen diferencias significativas. En cuanto a la flexo-extensión de codos y el salto largo a pies juntos, se observó que los estudiantes de la cohorte 2010 son quienes alcanzan los mejores registros de la muestra, seguidos por la cohorte 2009, 2013, 2011 y 2012, respectivamente. En relación al test de Course Navette, los datos indican que los mejores resultados fueron alcanzados por la cohorte 2012, luego las 2010, 2013, 2011 y 2009, respectivamente.

Al realizar las correlaciones, en los hombres UA se observó (tabla 4) que la ingesta de lácteos y la edad presentaron una correlación significativa, mientras que en mujeres UA las correlaciones se evidenciaron entre talla y menor consumo de frutas y bocadillos dulces, y perímetro de cintura. Por otra parte al correlacionar datos antropométricos y edad con las pruebas de condición física en hombres UA la edad, peso y perímetro de cintura e IMC se correlacionaron positivamente con la RCE ( $r=0,343 ; r=0,498 ; r=0,959$ y $r=0,608 ; p<0,001$ respectivamente), talla positivamente con salto largo a pies juntos $(r=0,391 ; p<0,001)$.

En el caso de las mujeres UA sólo existió una correlación negativa entre consumo de alimentos integrales y frituras $(r=-0,276 ; p<0,04)$.

En cuanto a la frecuencia en el consumo de alimentos por parte de los estudiantes UA (tabla 5), se observó un bajo cumplimiento de las porciones recomendadas, especialmente en lácteos descremados, frutas, pescados y leguminosas, por el contrario se apreció una elevada ingesta de sal y bebidas azucaradas, este último principalmente en hombres UA.

\section{DISCUSIÓN}

Diversos estudios en universitarios señalan que los estudiantes tienden a elegir alimentos ricos en lípidos, carbohidratos y bajos en fibra dietaria (35-37). Un estudio realizado por Durán y cols. (36) muestra un bajo consumo de frutas y verduras, situación similar a la presentada en otro estudio por españoles donde $84,9 \%$ no llegaba a las recomendaciones alimentarias (38), alcanzando sólo 4,7\% de ellos la ingesta sugerida de frutas. Por otra parte, Olivares y cols. (39) reportaron que las principales barreras en universitarios para no consumir frutas y verduras son "me da flojera prepararlas" o "se me olvida comerlas", tanto en hombres como en mujeres. Finalmente,

TABLA 4

Correlación entre alimentación y antropometría de los estudiantes de Educación Física, según sexo.

\begin{tabular}{|c|c|c|c|c|c|c|c|c|c|c|}
\hline & \multicolumn{5}{|c|}{ Hombres } & \multicolumn{5}{|c|}{ Mujeres } \\
\hline & Peso & Talla & PC & IMC & Edad & Peso & Talla & PC & IMC & Edad \\
\hline Desayuno & $-0,06$ & 0,09 & $-0,09$ & $-0,13$ & $-0,11$ & $-0,22$ & $-0,11$ & 0,25 & $-0,18$ & 0,07 \\
\hline Lácteos descremados & $-0,04$ & $-0,01$ & $-0,03$ & $-0,04$ & $0,21^{*}$ & $-0,21$ & $-0,07$ & $-0,15$ & $-0,19$ & $-0,02$ \\
\hline Frutas & 0,05 & $-0,02$ & 0,07 & 0,07 & $-0,05$ & $-0,08$ & $-0,26^{*}$ & $-0,02$ & 0,04 & $-0,02$ \\
\hline Verduras & $-0,07$ & 0,02 & $-0,03$ & $-0,10$ & $-0,01$ & $-0,17$ & $-0,16$ & 0,07 & $-0,10$ & $-0,01$ \\
\hline Pescado & 0,01 & $-0,02$ & 0,04 & 0,01 & 0,10 & $-0,13$ & 0,03 & $-0,03$ & $-0,16$ & $-0,01$ \\
\hline Leguminosas & $-0,02$ & 0,02 & 0,05 & $-0,04$ & 0,03 & 0,05 & 0,10 & $-0,04$ & 0,01 & 0,01 \\
\hline Alimentos integrales & $-0,04$ & $-0,03$ & $-0,02$ & $-0,03$ & $-0,01$ & $-0,24$ & $-0,30$ & $-0,06$ & $-0,11$ & $-0,01$ \\
\hline Comida casera & 0,03 & $-0,01$ & 0,11 & 0,05 & 0,10 & 0,05 & 0,07 & 0,05 & 0,02 & 0,08 \\
\hline Cena & $-0,09$ & $-0,04$ & $-0,10$ & $-0,08$ & $-0,03$ & $-0,12$ & $-0,22$ & $-0,18$ & $-0,02$ & 0,01 \\
\hline Bebidas azucaradas & 0,06 & 0,01 & 0,02 & 0,08 & $-0,02$ & $-0,13$ & 0,16 & $-0,26^{*}$ & $-0,24$ & $-0,08$ \\
\hline Alcohol & 0,05 & 0,04 & 0,05 & 0,05 & $-0,03$ & $-0,12$ & $-0,25$ & 0,04 & 0,01 & 0,03 \\
\hline Frituras & $-0,06$ & $-0,15$ & $-0,07$ & $-0,01$ & $-0,02$ & 0,01 & 0,18 & 0,07 & $-0,07$ & $-0,16$ \\
\hline Sal & 0,01 & 0,01 & $-0,02$ & 0,01 & $-0,01$ & 0,05 & 0,12 & 0,08 & $-0,01$ & $-0,29^{*}$ \\
\hline Comida rápida & $-0,06$ & $-0,07$ & $-0,04$ & $-0,04$ & $-0,05$ & 0,02 & $-0,09$ & 0,08 & 0,08 & 0,01 \\
\hline Bocadillos dulces & $-0,09$ & $-0,05$ & 0,01 & $-0,08$ & $-0,03$ & $-0,16$ & $-0,35^{* *}$ & $-0,12$ & $-0,01$ & $-0,01$ \\
\hline
\end{tabular}

Correlación de parcial de Pearson, $\mathrm{P}^{*}<0,05^{* *}<0,01 ; \mathrm{PC}=$ Perímetro de cintura; $\mathrm{IMC}=$ Índice de masa corporal. 
Rodríguez y Cols. (12) indican que en universitarios el nivel socioeconómico no tiene relación con los hábitos alimentarios.

Un estudio realizado en estudiantes universitarios chilenos (36) muestra que el consumo de cena incrementa de forma significativa la ingesta de frutas y verduras. En este estudio no se observó un consumo inferior a 2 comidas; estudios en universitarios japoneses revelaron que $81 \%$ consume 3 comidas, mientras que $59,8 \%$ de los estudiantes coreanos consume sólo 2 comidas al día (35). Respecto al desayuno nuestro estudio reveló que sólo 30\% de los estudiantes UA toma desayuno diariamente, similar a lo reportado para estudiantes coreanos $(32,6 \%)$ (35). Saltarse el desayuno se ha asociado con un peor estado nutricional y un mayor riesgo de enfermedad cardiovascular (36).

La mayoría de los estudiantes UA, independiente del sexo, al igual que otros estudios (36-40) ingieren alimentos de alta densidad energética, ricos en grasa saturada, azúcar y sal, que contribuyen a la ingesta calórica total de forma importante.

El estudio realizado por Espinoza y cols. (37) en estudiantes universitarios chilenos expone que las mujeres prefieren las golosinas en cambio los hombres prefieren la comida rápida, situación similar a lo reflejado en mujeres de la UA.

El estudio realizado por Troncoso y cols. (42) muestra que la familia influye positivamente en las conductas alimentarias saludables, en cambio los amigos y el plantel universitario lo hacen de forma negativa, principalmente por los horarios de clases que interfieren con las comidas.
En relación a los valores de IMC de los estudiantes UA, alcanzaron $64,3 \%$ de normopeso y $35,6 \%$ de sobrepeso y obesidad, puntajes deficientes al relacionarlos con los encontrados por Aránguiz y cols. (6), quienes evaluaron a estudiantes de las Universidades de Playa Ancha y Concepción en Chile, en donde $78,6 \%$ obtuvo rangos normales y sólo un 12\% registró sobrepeso. Al comparar los resultados por cohorte, se puede advertir que los estudiantes UA matriculados en 2013 y 2012 respectivamente, obtienen los mejores valores de IMC encontrándose $74 \%$ de normopeso, mientras que los alumnos (as) UA de cursos superiores (cohortes 2010 y 2009) alcanzan los registros más elevados de sobrepeso y obesidad $(46,7 \%)$. En consecuencia los valores promedio obtenidos por los estudiantes UA se acercan al límite superior de la normalidad, tendencia que aumenta al pasar los años alcanzando mayores puntajes de sobrepeso.

En relación al consumo de oxígeno (test de Course Navette) los varones UA obtuvieron mejores registros $(8,7 \pm 1,6$ minutos) frente a las mujeres $U A(5,4 \pm 1,8$ minutos), mientras que al observar los puntajes por año de ingreso la cohorte 2012 (6,8 \pm 1,8 minutos) es la más destacada. Al relacionar estos resultados con otros trabajos, no se encuentró información que haya evaluado universitarios con la misma prueba, sin embargo en el estudio realizado por Aránguiz y cols. (6) se analiza el mismo aspecto utilizando la caminata (2000 m), encontrando que 42,2\% alcanzaba el nivel aceptable. Cabe destacar que éstos últimos son universitarios de diversas ca-

\section{TABLA 5}

Frecuencia de consumo (\%) de algunos alimentos de los estudiantes de Educación Física, distribuidos por sexo.

\begin{tabular}{|c|c|c|c|c|c|c|}
\hline & \multicolumn{3}{|c|}{ Hombres } & \multicolumn{3}{|c|}{ Mujeres } \\
\hline & $\begin{array}{l}\text { No consume/ } \\
\text { nunca }\end{array}$ & $\begin{array}{l}\text { Ocasional/ } \\
\text { menos de lo } \\
\text { recomendado }\end{array}$ & $\begin{array}{c}\text { Todos los días/ } \\
\text { porciones } \\
\text { ideales al día }\end{array}$ & $\begin{array}{l}\text { No consume/ } \\
\text { nunca }\end{array}$ & $\begin{array}{l}\text { Ocasional/ } \\
\text { menos de lo } \\
\text { recomendado }\end{array}$ & $\begin{array}{c}\text { Todos los días/ } \\
\text { porciones } \\
\text { ideales al día }\end{array}$ \\
\hline Consumo de desayuno & 11,0 & 60,7 & 28,3 & 17,5 & 44,0 & 38,5 \\
\hline Lácteos descremados & 10,8 & 86,2 & 3,0 & 26,3 & 72,0 & 1,7 \\
\hline Frutas & 8,4 & 86,2 & 5,4 & 5,2 & 91,3 & 3,5 \\
\hline Verduras & 6,0 & 83,8 & 10,2 & 1,7 & 80,8 & 17,5 \\
\hline Pescado & 13,5 & 79,9 & 6,6 & 14,0 & 82,5 & 3,5 \\
\hline Leguminosas & 12,5 & 80,3 & 7,2 & 12,2 & 86,1 & 1,7 \\
\hline Alimentos integrales & 34,3 & 62,7 & 3,0 & 38,5 & 52,8 & 8,7 \\
\hline Comida casera & 4,8 & 48,4 & 46,8 & 3,5 & 45,7 & 50,8 \\
\hline \multirow[t]{2}{*}{ Consumo de cena } & 18,0 & 60,2 & 21,8 & 38,5 & 47,5 & 14,0 \\
\hline & $\begin{array}{l}\text { No consume/ } \\
\text { nunca }\end{array}$ & $\begin{array}{l}\text { Menos de } 1 \\
\text { o hasta } 2 \\
\text { porciones día }\end{array}$ & $\begin{array}{l}\text { Siempre/ } \\
3 \text { o más } \\
\text { porciones/día }\end{array}$ & $\begin{array}{l}\text { No consume/ } \\
\text { nunca }\end{array}$ & $\begin{array}{l}\text { Menos de } 1 \\
\text { o hasta } 2 \\
\text { porciones día }\end{array}$ & $\begin{array}{l}\text { Siempre/ } \\
3 \text { o más } \\
\text { porciones/día }\end{array}$ \\
\hline Bebidas azucaradas & 4,8 & 71,8 & 23,4 & 8,7 & 76,6 & 14,7 \\
\hline Alcohol & 32,5 & 55,5 & 12,0 & 40,3 & 56,2 & 3,5 \\
\hline Frituras & 39,9 & 46,9 & 13,2 & 5,3 & 76,2 & 18,5 \\
\hline Sal & 45,7 & 32,9 & 21,6 & 45,6 & 35,2 & 19,2 \\
\hline Comida rápida & 9,0 & 90,4 & 0,6 & 14,0 & 82,5 & 3,5 \\
\hline bocadillos dulces & 21,0 & 77,2 & 1,8 & 5,2 & 91,3 & 3,5 \\
\hline
\end{tabular}


rreras, mientras que los estudiantes UA pertenecen a la carrera de Pedagogía en Educación Física, sujetos con mayor nivel de actividad física lo que indica un mejor volumen de oxígeno máximo (VO2 max.) tal como lo mencionan López y cols. (33), quienes establecen que el entrenamiento físico puede inducir aumentos sustanciales en el valor del VO2 máx.

En cuanto a las pruebas de flexo-extensión de codos, salto largo a pies juntos y abdominales cortos las mujeres UA obtuvieron puntajes más bajos que respecto los varones UA, siendo superiores sólo en la prueba de flexibilidad. Al analizar los resultados por cohorte se puede apreciar que para la prueba de abdominales cortos sólo las cohortes 2009 y 2010 logran completar la prueba, en el caso del salto largo a pies juntos quienes obtienen los mejores puntajes son los estudiantes UA 2010 y en cuanto a la flexibilidad el grupo 2013 son los más destacados.

$\mathrm{Al}$ indagar en trabajos que expongan resultados similares a los presentados, sólo se encuentra el SIMCE 2012 realizado en Chile para estudiantes de $8^{\circ}$ básico, en el cual se observó que sólo $8 \%$ de la muestra alcanza el nivel satisfactorio. Diferencias que pueden ser explicadas por la mayor cantidad de masa muscular de los universitarios, lo que conlleva a un mejor rendimiento en las pruebas realizadas, lo que se respalda con lo afirmado por López y cols. (33), quienes indican "Los músculos son los responsables del movimiento, la proporción del tejido muscular con relación al peso corporal aumenta, hasta alcanzar $50 \%$ o más en la edad adulta".

\section{CONCLUSIÓN}

Los resultados obtenidos en esta investigación reflejan un mejor rendimiento físico de los estudiantes UA de las cohortes 2010 y 2012, situación que podría explicarse por la mayor cantidad de asignaturas prácticas, dado que al analizar la malla curricular de Pedagogía en Educación Física, se advierte que los estudiantes UA de cuarto (cohorte 2010) y segundo año (cohorte 2012) ostentan la mayor carga académica en actividades prácticas con 9 y 7 asignaturas respectivamente que incluyen actividad física, mientras que el resto de las cohortes alcanza entre 4 y 5 asignaturas por año.

En síntesis, los estudiantes UA presentan malos hábitos alimentarios durante toda la carrera universitaria, gran parte de ellos presentan sobrepeso u obesidad, mientras que su condición física disminuye de manera irregular a medida que avanzan los estudios, situación preocupante ya que se transformarán en modelos de estilos de vida saludable especialmente a nivel escolar.

\section{RESUMEN}

Objetivo: Determinar los hábitos alimentarios y condición física de los estudiantes de pedagogía en educación física de la Universidad Autónoma de Chile, sede Temuco y relacionarlos con el nivel de condición física. Sujetos y métodos: Estudio transversal observacional, se evaluaron 239 estudiantes de educación física, de los cuales 76,5\% eran hombres, aplicándoles una encuesta alimentaria, antropometría y evaluación de la condición física. Resultados: El 35,6\% de los estudiantes presentaba sobrepeso/obesidad, además tenían malos hábitos alimentarios independientes del año de ingreso. Sólo 4,7\% consumía las recomendaciones de frutas y 30\% tomaba desayuno todos los días. En hombres el peso, la circunferencia de la cintura e IMC correlacionan negativamente con la prueba de Course navette $(R=-0,203 ; R=-0,249 ; R=-0,196, p<0,01$ respectivamente). Conclusión: Los estudiantes universitarios presentan malos hábitos alimentarios durante toda la carrera universitaria, mientras que su condición física disminuye de manera irregular a medida que avanzan los estudios, situación preocupante ya que ellos se transformaran en modelos de estilos de vida saludable especialmente a nivel escolar.

Palabras clave: Estudiante universitario, antropometría, condición física, encuesta alimentaria.

\section{BIBLIOGRAFÍA}

1. Sumalla S, Elío I, Domínguez I, Calderón $R$, García Á, Fernández F, Gracia S, Dzul L, Battino M, Solano H. Valoración del perfil e ingesta de nutrientes de un grupo de estudiantes iberoamericanos de postgrados en nutrición. Nutr Hosp. 2013; 28:532-40.

2. Montero A, Úbeda N, García A. Evaluación de los hábitos alimentarios de una población de estudiantes universitarios en relación con sus conocimientos nutricionales. Nutr Hosp. 2006; 21:466-73.

3. Cervera F, Serrano R, García C, Milla M, García MJ. Hábitos alimentarios y evaluación nutricional en una población universitaria. Nutr Hosp. 2013; 28:438-46.

4. Universidad de Concepción. Encuesta nacional de hábitos de actividad física y deportes en la población Chilena de 18 años y más. Facultad de Ciencias Económicas y Administrativas. Universidad de Concepción. Concepción, Chile. 2012.

5. MINEDUC. SIMCE 2012 Educación Física, resultados para Docentes y Directivos. Santiago: Ministerio de Educación, 2013.

6. Aránguiz H, García V, Rojas S, Salas C, Martínez $R$, Mac Millan N. Estudio descriptivo, comparativo y correlacional del estado nutricional y condición cardiorrespiratoria en estudiantes universitarios de Chile. Rev Chil Nutr. 2010; 37:70-8.

7. Barragán M. Hábitos alimentarios en estudiantes de la Universidad Juárez Autónoma de Tabasco. Rev Cubana Salud Pública 2006; 32:911-22.

8. Iglesias M, Mata $G$, Pérez A, Hernández S, García R, Papadaki $C$. Estudio nutricional en un grupo de estudiantes universitarios Madrileños. Nutr Clín Diet Hosp. 2013; 33:23-30.

9. Jáuregui-Lobera I. Conocimientos, actitudes y conductas: hábitos alimentarios en un grupo de estudiantes de nutrición. Trastornos Conducta Alim. 2010; 11:1183-95.

10. Ratner $R$, Hernández P, Martel J, Atalah E. Calidad de la alimentación y estado nutricional en estudiantes universitarios de 11 regiones de Chile. Rev Méd Chil. 2012; 140:1571-9.

11. Troncoso C, Doepking C, Zúñiga C. Alimentación saludable en la formación de estudiantes de carreras del área pedagógica. Rev Chil Nutr. 2013; 40:43-7.

12. Rodríguez F, Palma X, Romo Á, Escobar D, Aragú B, Espinoza L, McMillan N, Gálvez J. Hábitos alimentarios, actividad física y nivel socioeconómico en estudiantes universitarios de Chile. Nutr Hosp. 2013; 28:447-55.

13. Almagià $A$, Lizana $P$, Rodríguez $F$, Ivanovic $D$, Binvignat $G$. Variables Antropométricas y Rendimiento Físico en Estudiantes Universitarios de Educación Física. Int J Morphol. 2009; 27:971-5.

14. García J, Alonso D. Valoración de la condición física saludable en Universitarios Gallegos. Rev Int Med Cienc Act Fís Deporte 2010; 11:781-90.

15. Martínez L. Condición física y nivel de actividad física en estudiantes universitarios. Rev Teoría y Praxis Invest. 2008; 3:21-8.

16. Valdés-Badilla P, Godoy A, Caniuqueo A. Medición de la Condición Física de los Estudiantes de Pedagogía en Edu- 
cación Física, utilizando el Simce de la Especialidad. Rev Ciencias Actividad Física UCM 2013; 14:21-9.

17. Valdés-Badilla P, Godoy A, Gedda R. Comparación por cohorte de la condición física de estudiantes de pedagogía en educación física de la Universidad Autónoma de Chile, sede Temuco. Rev Horiz Cienc Act. Fís. 2013; 6:76-85.

18. MINEDUC. Bases curriculares 2013, Educación Física y Salud. Santiago: Ministerio de Educación de Chile. 2013.

19. Linstone HA, Turoff $M$. The Delphi method: Techniques and applications. Reading, MA: Addison WEsley Publishing 1975

20. Canadian Society for Exercise Physiology (CSEP). The Canadian Physical Activity, Fitness and Lifestyle Approach: CSEP-Health \& Fitness Program's Health-Related Appraisal and Counselling Strategy. Canadian Society for Exercise Physiology., Ottawa, 2003.

21. Gadoury C, Leger L. Validite de l'epreuve de course navette de $20 \mathrm{~m}$ avec paliers de une minute et du physitest canadien pour predire le VO2 max des adultes. Revue Staps 1985; 13:57-68.

22. Gatica P. La condición física en la población escolar de la región del Maule-Chile. Talca: Universidad Católica del Maule, 2000.

23. Instituto Nacional de Deportes. Aplicación de Instrumentos de Medición de la Condición Física en Alumnos de Enseñanza Básica. Instituto Nacional de Deportes., Santiago, 2006.

24. Jódar R. Revisión de artículos sobre la validez de la prueba de Course Navette para determinar de manera indirecta el VO2 máx. Rev Int Med Cienc Act Fís Deporte 2003; 11:173-81.

25. Montecinos R. La aptitud física en la población chilena (Proyecto FONDECYT N 1970061). Universidad Católica del Maule. 2000.

26. Montecinos $R$, Gatica $P$, Trujillo $H$, Vargas $R$, Herrera $M$, Jirón O. (2005). Test para evaluar la condición física en escolares chilenos. Rev Arch Soc Chil Med Deporte 2005; 50: 9-24.

27. Montecinos $R$, Gatica P. Condición Física de la población escolar chilena femenina de 10 a 18 años de edad. Rev Arch Soc Chil Med Deporte. 2005; 50:125-40.

28. Tremblay M. Fitness of Canadian Children and Youth: Results from the 2007- 2009 Canadian Health Measures Survey. Statistics Canada, Health Reports, pp. www.statcan.gc.ca/pub/82-003-x/2010001/article/11065-eng. pdf. 2010.

29. Arnaiz P, Acevedo M, Díaz C, Bancalari R, Barja S, Aglony M, Cavada G, García H. Razón cintura estatura como predictor de riesgo cardiometabólico en niños. Rev Chil Cardiol 2010; 29:281-8.

30. Lamela M. Valoración de la Condición Física relacionada con la salud en el ámbito educativo. Recuperado el 20 de diciembre de 2012, de Valoración de la Condición Física relacionada con la salud en el ámbito educativo: http:// www.edu.xunta.es/centros/cfrlugo/system/files/valorac i\%c3\%B3ncondici\%C3\%B3nf\%C3\%ADsica.pdf. 2009.

31. Nogueira J. Valoración de la condición física en niños de 11-12 años con distinto nivel socio-económico. Rev Int Med Cienc Act Fís Deporte 2002; 2:177-88.

32. Blázquez D. Evaluar en Educación Física. INDE, Barcelona, 2010.

33. López C, Fernández V. Fisiología del ejercicio. Panamericana, Madrid, 2006.

34. Martínez L, Ramírez F. Experiencias en Educación Física. Barcelona, Universidad de Barcelona, 2002.

35. Sakamaki R, Amamoto R, Mochida Y, Shinfuku N, Toyama $K$. A comparative study of food habits and body shape perception of university students in Japan and Korea Nutr J. 2005; 4:31-40.

36. Sakata $K$, Matumara $Y$, Yoshimura N, Tamaki J, Hashimoto T, Oguri S, Okayama A, Yanagawa H. Relationship between skipping breakfast and cardiovascular disease risk factors in the national nutrition survey data. Nipón Koshu Eisei Zasshi 2001; 48:837-41

37. Espinoza L, Rodríguez F, Gálvez J, MacMillan N. Hábitos de alimentación y actividad física. Rev Chil Nutr. 2011; 38:458-65.

38. Durán S, Castillo M, Vio F. Diferencias en la calidad de vida de estudiantes universitarios de diferente año de ingreso del Campus Antumapu. Rev Chil Nutr. 2009; 33:200-9.

39. Durán S, Bazaez G, Figueroa K, Berlanga MR, Encina C, Rodríguez MP. Comparación en calidad de vida y estado nutricional entre alumnos de nutrición y dietética y de otras carreras universitarias de la Universidad Santo Tomás de Chile. Nutr Hosp. 2012; 27:739-46.

40. Arroyo M, Rocandio AM, Ansotegui L, Pascual E, Salces I, Rebato E. Calidad de la dieta, sobrepeso y obesidad en estudiantes universitarios. Nutr Hosp. 2006; 21:673-9

41. Olivares S, Lera L, Bustos N. Etapas del cambio, beneficios y barreras en actividad física y consumo de frutas y verduras en estudiantes universitarios de Santiago de Chile. Rev Chil Nutr. 2008; 35:25-35.

42. Troncoso C, Amaya J. Factores sociales en las conductas alimentarias de estudiantes universitarios. Rev Chil Nutr. 2009; 36:1090-7.

\section{ANEXO}

Nombre:

Edad:

Carrera:

Facultad:

Presentación o encabezado: estimado (a) estudiante: esta es una encuesta anónima cuyo propósito es. por tal motivo agradeceremos a Ud. etc.

I. Encierre en un círculo la alternativa que más se identifique con su consumo habitual de alimentos.

1. ¿Toma desayuno?
a) Nunca
b) menos de 1 vez a la semana
c) 1-3 veces por semana
d) 4-6 veces por semana
e) todos los días 
2. ¿Consume lácteos descremados (leche descremada, quesillo, queso fresco o yogur descremado)?
a) No consume
b) menos de 1 vez al día
c) 1 porción al día

d) 2 porciones día

e) 3 porciones día

3. Consume frutas (frescas de tamaño regular)
a) No consume
b) menos de 1 vez al día
c) 1 porción al día
d) 2 porciones día
e) 3 porciones día

4. Consume verduras (crudas o cocidas_porción equivalente a 1 plato servilleta)
a) No consume
b) menos de 1 vez al día
c) $1 / 2$ porción al día
d) 1 porciones día
e) 2 porciones día

5. Consume pescado (fresco/congelado/conserva_no frito)
a) no consume
b) menos de 1 vez a la semana
c) 1 porción semana
d) 2 porciones semana
e) 3 porciones semana

6. Consume leguminosas (porotos, lentejas, arvejas, garbanzos)
a) No consume
b) menos de 1 vez a la semana
c) 1 porción semana
d) 2 porciones semana
e) 3 o más porciones semana

7. consume avena o panes integrales
a) No consume
b) menos de 3 veces en la semana
c) 1 porción al día
d) 2 porciones día
e) 3 porciones día

8. usted come comida del hogar (casera: guisos, carbonadas, cazuela, legumbres)
a) No consume
b) menos de 1 vez a la semana
c) 1 porción semana
d) 2 porciones semana
e) 3 o más porciones semana

9. usted cena (comida + fruta y/o ensalada)
a) Nunca
b) menos de 1 vez a la semana
c) 1-3 veces por semana
d) 4-6 veces por semana
e) todos los días

II. Encierre en un círculo la alternativa que más se identifique con su consumo habitual.

1. Toma bebidas o jugos azucarados (porción 1 vaso de 200cc)
a) No consume
b) menos de 1 vez al día
c) 1 porción al día
d) 2 porciones día
e) 3 porciones día

2. Consume bebidas alcohólicas (porción 1 vaso) viernes o sábado
a) No consume
b) menos de 1 vez al día
c) 1 porción al día
d) 2 porciones día
e) 3 porciones día

\section{Consume frituras}
a) No consume
b) ocasionalmente

c) 1 porción semana

d) 2 porciones semana

e) 3porciones semana

4. Usted le agrega sal a las comidas antes de probarlas
a) No le agrega
b) le agrega ocasionalmente
c) siempre le agrega

5. Consume pizza, completos, hamburguesas, papas fritas, sopaipillas.
a) No consume
b) menos de 1 vez al día
c) 1 porción al día

d) 2 porciones día

e) 3 porciones día

6. Consume en la Universidad como colación galletas y snack dulces
a) No consume
b) menos de 1 vez al día
c) 1 porción al día
d) 2 porciones día
e) 3 porciones día

\section{GRACIAS POR SU TIEMPO!}

Nombre del responsable o entidad encargada del estudio. 